\title{
ALGORITMO PREDITIVO BASEADO EM MODELO APLICADO AO CONTROLE DE VELOCIDADE DO MOTOR DE INDUÇÃO
}

\author{
Eudemario S. de Santana* \\ eudemario@gmail.com
}

\author{
Edson Bim* \\ bimedsce. fee. uni camp.br
}

\author{
Wagner C. Amaral* \\ wagneredca.fee.unicamp.br
}

*Universidade Estadual de Campinas, FEEC

Av. Albert Einstein, $\mathrm{n}^{\circ} 400$, Cidade Universitária Zeferino Vaz, Campinas SP

\begin{abstract}
This article presents the results concerning the control of rotor flux and speed of the induction motor using MBPC algorithm. A nonlinear state space model of induction motor is used to describe its dynamic. The rotor flux and speed are estimated using an extended Kalman filter. The load torque is considered as a disturbance and its value is calculated through the electromechanical equation. Simulated and experimental results have shown the good performance of this strategy.
\end{abstract}

KEYWORDS: Induction motor, model based predictive control, field orientation.

\section{RESUMO}

Este artigo trata do emprego de um algoritmo preditivo no controle do fluxo do rotor e da velocidade de um motor de indução trifásico. No acionamento elétrico proposto, o motor de indução (MI) é descrito em espaço de estados, sendo necessário, para a realização do controle, o conhecimento de suas tensões de terminal do estator e das seguintes variáveis de estado: corrente de estator, fluxo de rotor e velocidade de eixo do motor, sendo que estas duas últimas são estimadas por um filtro de Kalman estendido. O torque de carga é

Artigo submetido em 16/02/2006

1a. Revisão em 01/06/2006

2a. Revisão em 26/10/2007

Aceito sob recomendação do Editor Associado Prof. José Antenor Pomilio considerado uma perturbação e a sua amplitude é calculada a partir do emprego da equação eletromecânica. A aplicação do MBPC é mostrada por resultados de simulação computacional e experimentais.

PALAVRAS-CHAVE: Motor de indução, controle preditivo baseado em modelo, orientação de fluxo.

\section{LISTA DE SÍMBOLOS}

$L, \tau \quad$ indutância e constante de tempo

$L^{\prime}, \tau^{\prime} \quad$ indutância e constante de tempo transitórias

$R \quad$ resistência elétrica

$J_{t} \quad$ momento de inércia total das partes girantes

$i, V, \Phi$ corrente, tensão e fluxo concatenado

$\omega$ freqüência elétrica ou velocidade angular

$p \quad$ número de pares de pólos

$d, q \quad$ eixos direto e em quadratura

$e, t \quad$ escorregamento, total

$m$ magnetização ou motor

$s, r, c \quad$ estator, rotor, carga

$T_{a} \quad$ tempo de amostragem

\section{INTRODUÇÃO}

A maioria das estratégias de controle empregadas para acionamentos elétricos com o motor de indução (MI) utiliza controladores com uma entrada e uma saída (SISO - Single-input Single-output) para controlar com malhas distintas o fluxo e a velocidade, o que significa que uma entrada específica 
relaciona-se exclusivamente com uma saída específica, isto é, supõe-se que o sistema é desacoplado. Esta suposição não é verdadeira para o acionamento do MI alimentado por um inversor fonte de tensão, pois as tensões de eixos direto e em quadratura influenciam simultaneamente o fluxo - seja o de estator, o de entreferro ou o de rotor - e a velocidade. Assim, neste trabalho utiliza-se um controlador multivariável que considera as interações existentes entre as variáveis do motor de indução.

Dentre as diversas estratégias existentes de controle multivariável, tem-se destacado a do controle preditivo baseado em modelo (MBPC - Model Based Predictive Control), ou apenas controle preditivo. O MBPC é uma classe de técnicas de controle que utilizam um modelo do processo para prever seu comportamento futuro. A lei de controle dessa estratégia é obtida a partir da otimização de uma função, que considera o esforço de controle necessário para atuar no sistema, e o erro entre a saída prevista e a referência especificada. De acordo com a estratégia de horizonte deslizante, aplica-se apenas o primeiro elemento da sequência ótima de controle ao processo. No instante seguinte da amostragem, novas medições são realizadas na planta e os procedimentos de previsão e de otimização são repetidos (Moreira. V.D. (2004)). Se o modelo empregado para realizar a predição é não linear, a otimização exige métodos numéricos. No trabalho relatado neste artigo, realizam-se linearizações sucessivas em cada instante de amostragem para se obter uma expressão analítica para a lei de controle.

$\mathrm{Na}$ literatura são relatadas algumas aplicações do MBPC ao acionamento de máquinas elétricas rotativas, como é o caso de o controle de posição de motores brushless $D C$, implementado experimentalmente e com sucesso por Low et al. (1997): os resultados obtidos mostram que o desempenho do sistema fica dentro das especificações desejadas, ainda que o torque de carga e a inércia variem. Uma aplicação do algoritmo MBPC ao acionamento do motor de indução foi proposto por Zhang et al. (1997), sendo apresentados apenas resultados de simulação computacional relativos ao controle da corrente do estator. Kennel et al. (1997) utilizam uma técnica MBPC intitulada Controle Pre-ditivo Generalizado (GPC- Generalizedodel Predictive Control) na implementação de um controlador de cor-rente, a partir de um modelo linear da equação de tensões de estator da máquina de indução. Os autores utilizam vários controladores preditivos generalizados SISO, sendo que há um para controle do componente de eixo direto da corrente de estator, que tem como saída a tensão de eixo direto de estator, e dois outros controladores GPC em cascata. Um destes controladores gera o componente de eixo em quadratura da tensão de estator, a partir da referência do componente em quadratura da corrente, que, por sua vez, é gerado por outro GPC, que utiliza a referência de velocidade de eixo do rotor. Em Kennel and Linder (2005b) é apresentada uma estratégia que reduz a computação em tempo real de um controlador preditivo aplicado no controle de corrente de um motor de indução, sem que seja explicitado se é feito algum controle de velocidade. Os resultados experimentais, segundo os autores, mostram o potencial dos controladores preditivos baseados no modelo como uma alternativa aos clássicos PI. Em outro artigo, escrito por Kennel and Linder (2005a) usam um controle preditivo para controlar diretamente um inversor trifásico. Novamente, os resultados experimentais apresentados são apenas as componentes de eixo direto e em quadratura das correntes do estator, não deixando claro se é feito o controle de velocidade. Hedjar et al. (2004) apresentaram uma proposta na qual uma estrutura MBPC em cascata é empregada para controlar a velocidade e o torque do MI, sem considerar o torque de carga na modelagem.

Neste trabalho emprega-se um modelo de estado não linear. Utiliza-se ainda a orientação de fluxo do rotor, o que torna o fluxo do rotor inteiramente definido no eixo direto do sistema síncrono de coordenadas (Altuna, 2002). Desta forma, as variáveis de estado são os componentes de eixo direto e em quadratura da corrente de estator, o fluxo de rotor e a velocidade de eixo do motor. Geralmente, para tornar o acionamento mais robusto e barato, empregam-se estimadores de estado (fluxo e velocidade) como, por exemplo, o filtro de Kalman estendido (Valdenebro, 2001).

Este artigo trata da aplicação do controlador preditivo baseado no modelo linearizado a cada amostragem realizada no controle da velocidade e do fluxo de rotor do MI, sendo estas variáveis estimadas mediante um filtro de Kalman estendido (EKF). São apresentados resultados de simulação digital e de testes experimentais com o objetivo de validar a presente proposta. As equações do controle MBPC, a discretização e linearização do modelo do MI são apresentadas na seção 2, enquanto a descrição da estratégia da aplicação da estratégia MBPC linearizada ao acionamento do MI, considerando o torque de carga como uma perturbação, é o conteúdo da seção 3. Os resultados das simulações computacionais e dos testes experimentais estão colocados na seção 4 , para na seqüência, seção 5 , ter-se as conclusões.

\section{MODELO DO MOTOR DE INDUÇÃO EM ESPAÇO DE ESTADO}

O controle de alto desempenho de um motor de indução é realizado empregando a orientação do fluxo, sendo que a orientação do fluxo do rotor tem a vantagem de ser desacoplado: uma componente da corrente de estator é responsável pelo fluxo do rotor e outra está associada ao torque. Neste trabalho, então, faz-se o controle de velocidade de eixo do motor empregando a estratégia da orientação do fluxo de rotor. Sendo assim, a componente em quadratura do fluxo do rotor 


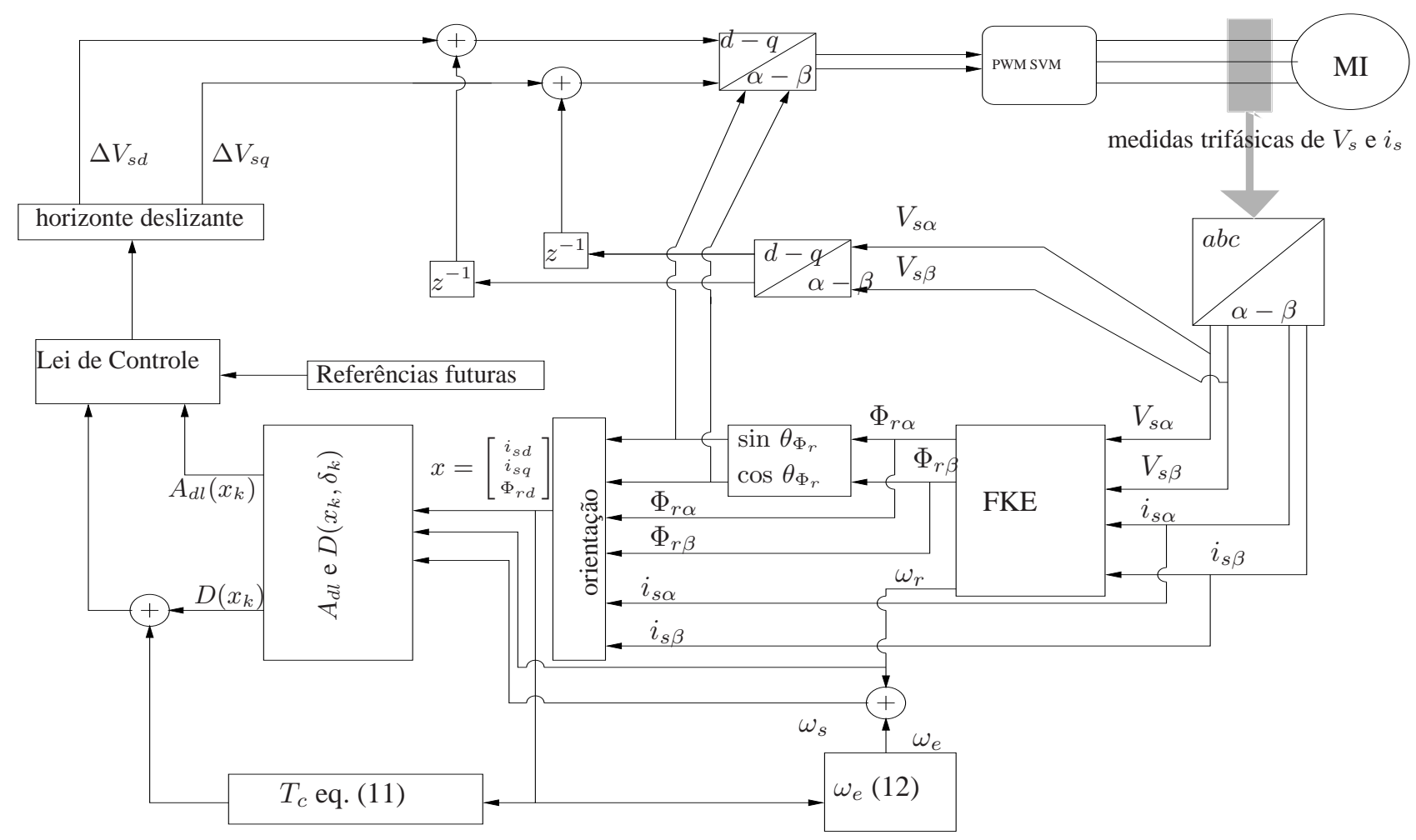

Figura 1: Diagrama de bloco representativo do acionamento proposto.

é nula e, portanto, tem-se $\Phi_{r}=\Phi_{r d}$.

O modelo do MI escrito em espaço de estados é dado por

$$
\left\{\begin{array}{l}
\dot{x}=f(x)+B u+\delta=A(x) x+B u+\delta \\
y=C x
\end{array}\right.
$$

na qual:

- $x \in \Re^{(4 \times 1)}$ é o vetor de estados descrito por $x=\left[\begin{array}{llll}i_{s d} & i_{s q} & \Phi_{r d} & \omega_{r}\end{array}\right]^{T} ;$

- $u \in \Re^{(2 \times 1)}$ é o vetor de entrada composto por tensões de terminal e descrito por $u=\left[\begin{array}{ll}V_{s d} & V_{s q}\end{array}\right]^{T}$;

- $\delta \in \Re^{(4 \times 1)}$ é o vetor de perturbação e dado por $\delta=$ $\left[\begin{array}{llll}0 & 0 & 0 & \left(-p / J_{t}\right) T_{c}\end{array}\right]^{T}$;

- $f(x)$ é a função não-linear, que é desmembrada em $A(x) x$, sendo que a matriz característica do sistema
$A(x) \in \Re^{(4 \times 4)}$ é dada por

$$
A=\left[\begin{array}{cccc}
\frac{-1}{\tau_{s}^{\prime}} & \omega_{s} & \frac{L_{m}}{L_{s}^{\prime} L_{r} \tau_{r}} & 0 \\
-\omega_{s} & \frac{-1}{\tau_{s}^{\prime}} & -\frac{\omega_{r} L_{m}}{L_{s}^{\prime} L_{r}} & 0 \\
\frac{L_{m}}{\tau_{r}} & 0 & \frac{-1}{\tau_{r}} & 0 \\
0 & \kappa \Phi_{r d} & 0 & 0
\end{array}\right]
$$

na qual $\kappa=(3 / 2)\left(p / J_{t}\right)\left(L_{m} / L_{r}\right), L_{s}^{\prime}$ é a indutância transitória do estator, $\tau_{s}^{\prime}=L_{s}^{\prime} / R_{s}$ é a constante de tempo transitória do estator e $\tau_{r}=L_{r} / R_{r}$ é a constante de tempo do rotor.

- $B \in \Re^{(4 \times 2)}$ é a matriz de entrada, dada por

$$
B=\left[\begin{array}{cc}
1 / L_{s}^{\prime} & 0 \\
0 & 1 / L_{s}^{\prime} \\
0 & 0 \\
0 & 0
\end{array}\right]
$$

- $y \in \Re^{(2 \times 1)}$ é o vetor de saída dado por $y=\left[\begin{array}{ll}\Phi_{r d} & \omega_{r}\end{array}\right]^{T}$; 
- A matriz de saída $C \in \Re^{(2 \times 4)}$ e é dada por

$$
C=\left[\begin{array}{llll}
0 & 0 & 1 & 0 \\
0 & 0 & 0 & 1
\end{array}\right]
$$

O modelo espaço-estado na forma discreta é dado por

$$
\left\{\begin{array}{l}
x_{k+1}=A_{d l}(x) x_{k}+B_{d} u_{k}+D\left(x_{k}, \delta_{k}\right) \\
y_{k+1}=C_{d} x_{k+1}
\end{array}\right.
$$

na qual $A_{d}, B_{d}$ e $C_{d}$ representam respectivamente as formas discretas das matrizes $A, B$ e $C$. Estas matrizes são avaliadas a partir da expansão em séries de segunda ordem da matriz exponencial como segue:

$$
\left\{\begin{array}{l}
A_{d}=e^{A T_{a}} \approx I+A T_{a}+\frac{A^{2} T_{a}^{2}}{2} \\
B_{d}=\int_{0}^{\tau} e^{A T_{a}} B d \tau \approx B T_{a}+\frac{A B T_{a}^{2}}{2} \\
C_{d}=C
\end{array}\right.
$$

nas quais $T_{a}$ representa o período de amostragem.

Se no instante de amostragem $k$ os estados são conhecidos e representados por $\widehat{x}_{k}$, a matriz do sistema para o caso discreto (equação (3)) é linearizada empregando a seguinte operação:

$$
A_{d l}=\partial\left[A_{d}\left(x_{k}\right) x_{k}\right] /\left.\partial x_{k}\right|_{x_{k}}
$$

Este procedimento gera um termo extra $\left[f\left(\widehat{x}_{k}\right)-A\left(\widehat{x}_{k}\right) \widehat{x}_{k}\right]$, que somado à $\delta_{k}$ resulta em $D\left(x_{k}, \delta_{k}\right)$. Ainda que a perturbação seja nula - no nosso caso esta pertubação é dada no torque de carga $\left(T_{c}\right)$-, há um termo adicional na equação (3) devido à linearização do modelo. No caso do MI, têm-se as seguintes matrizes:

$$
A_{d l}=\left[\begin{array}{cccc}
1-\frac{T_{a}}{\tau_{s}^{\prime}} & T_{a} \omega_{s} & \frac{T_{a} L_{m}}{L_{s}^{\prime} L_{r} \tau_{r}} & 0 \\
-T_{a} \omega_{s} & 1-\frac{T_{a}}{\tau_{s}^{\prime}} & -\frac{T_{a} \widehat{\omega}_{r} L_{m}}{L_{s}^{\prime} L_{r}} & -\frac{T_{a} L_{m} \widehat{\Phi}_{r d}}{L_{s}^{\prime} L_{r}} \\
\frac{T_{a} L_{m}}{\tau_{r}} & 0 & 1-\frac{T_{a}}{\tau_{r}} & 0 \\
0 & \kappa T_{a} \widehat{\Phi}_{r d} & T_{a} \kappa \widehat{i}_{s q} & 1
\end{array}\right]
$$

e o vetor $D\left(x_{k}, \delta_{k}\right)$ é

$$
D\left(x_{k}, \delta_{k}\right)=\left[\begin{array}{c}
0 \\
\frac{T_{a} L_{m} \widehat{\Phi}_{r d} \widehat{\omega}_{r}}{L_{s}^{\prime} L_{r}} \\
0 \\
-\kappa T_{a} \widehat{i}_{s q} \widehat{\Phi}_{r d}-\frac{p}{J_{t}} T_{c}
\end{array}\right]
$$

\section{CONTROLE PREDITIVO DO MOTOR DE INDUÇÃO}

O algoritmo de controle preditivo é constituído essencialmente pelas etapas da predição e da determinação da lei de controle, como descritas na seqüência.

\subsection{Predição}

Nesta etapa encontram-se as maiores diferença das técnicas usadas no MBPC. Com o intuito de reduzir o erro de regime permanente das saídas do processo, emprega-se um modelo incremental que utiliza o seguinte modelo de espaço de estado aumentado (Rossiter, 2003):

$$
\left\{\begin{array}{l}
\mathbf{x}_{k+1}=\tilde{A} \mathbf{x}_{k}+\tilde{B} \Delta u_{k}+\tilde{D} \\
y_{k+1}=\tilde{C} \mathbf{x}_{k+1}
\end{array}\right.
$$

na qual

$$
\begin{gathered}
\tilde{A}=\left[\begin{array}{cc}
A_{d l}(x) & B_{d} \\
0_{p \times n} & I_{p \times p}
\end{array}\right] \quad \tilde{B}=\left[\begin{array}{c}
B_{d} \\
I_{p \times p}
\end{array}\right] \\
\tilde{D}=\left[\begin{array}{c}
D \\
0_{p \times 1}
\end{array}\right] \quad \tilde{C}=\left[\begin{array}{ll}
C_{d} & 0_{q \times p}
\end{array}\right] \\
\mathbf{x}_{k}^{T}=\left[\begin{array}{ll}
x_{k}^{T} & u_{k-1}^{t}
\end{array}\right]
\end{gathered}
$$

Sendo $n$ o número de estados, $p$ o número de entradas e $q$ o número de saídas, tem-se que $\tilde{A} \in \Re^{[(n+p) \times(n+p)]}, \tilde{B} \in$ $\Re^{[(n+p) \times p]}, \tilde{D} \in \Re^{[(n+p) \times 1]}$ e $\tilde{C} \in \Re^{[q \times(n+p)]}$.

O conjunto de previsões é dado por

$$
\hat{\mathbf{y}}=H^{\prime} \mathbf{x}+H^{\prime \prime} \Delta \mathbf{u}+H^{\prime \prime \prime} \mathbf{d}
$$

na qual

- $\hat{\mathbf{y}} \in \Re^{\left(n_{y} q\right) \times 1}$ é o vetor predição da saída, com $n_{y}$ sendo o horizonte de predição da saída;

- $\mathbf{x} \in \Re^{n_{y} n+p}$ é o estado estimado por um filtro de Kalman;

- $\Delta \mathbf{u} \in \Re^{n_{u} p \times 1}$ é o vetor das entradas incrementais, com $n_{u}$ sendo o horizonte de controle;

- $\mathbf{d} \in \Re^{\left[\left(n_{y} n\right) \times 1\right]}$ é a predição do distúrbio;

- $H^{\prime} \in \Re^{\left[\left(n_{y} q\right) \times n\right]}, H^{\prime \prime} \in \Re^{\left[\left(n_{y} q\right) \times\left(n_{u} p\right)\right]}$

- e $H^{\prime \prime \prime} \in \Re^{\left[\left(n_{y} q\right) \times\left(n_{y} n\right)\right]}$ são as matrizes dadas por 


$$
\begin{gathered}
H^{\prime \prime}=\left[\begin{array}{ccccc}
\tilde{C} \tilde{B} & 0 & 0 & \cdots & 0 \\
\tilde{C} \tilde{A} \tilde{B} & \tilde{C} \tilde{B} & 0 & \cdots & 0 \\
\tilde{C} \tilde{A}^{2} \tilde{B} & \tilde{C} \tilde{A} \tilde{B} & \tilde{C} \tilde{B} & \cdots & 0 \\
\vdots & \vdots & \vdots & \ddots & \vdots \\
\tilde{C} \tilde{A}^{n_{y}-1} \tilde{B} & \tilde{C} \tilde{A}^{n y-2} \tilde{B} & \cdots & & \tilde{C} \tilde{B}
\end{array}\right] \\
H^{\prime \prime \prime}=\left[\begin{array}{ccccc}
\tilde{C} & 0 & 0 & \cdots & 0 \\
\tilde{C} \tilde{A} & \tilde{C} & 0 & \cdots & 0 \\
\tilde{C} \tilde{A}^{2} & \tilde{C} \tilde{A} & \tilde{C} & \cdots & 0 \\
\vdots & \vdots & \vdots & \ddots & \vdots \\
\tilde{C} \tilde{A}^{n_{y}-1} & \tilde{C} \tilde{A}^{n_{y}-2} & \tilde{C} \tilde{A}^{n_{y}-3} & \cdots & \tilde{C}
\end{array}\right]
\end{gathered}
$$

Na estimação dos estados do sistema, utilizou-se um EKF no sistema descrito pela equação (3), que representa adequadamente o processo em velocidades elevadas de eixo. Embora o controle proposto seja aplicado ao motor com orientação do fluxo de rotor, o que significa que o sistema de coordenadas gira na velocidade síncrona, a estimação é realizada com o emprego do modelo do motor descrito nas coordenadas estacionárias $\alpha-\beta$, porque, além deste fluxo independer do sistema de coordenadas, sua posição é facilmente determinada a partir dos valores instantâneos de $\Phi_{r \alpha}$ e $\Phi_{r \beta}$ que, por sua vez, são obtidos a partir das variáveis tensão e corrente medidas por sensores; uma vez determinados $\Phi_{r \alpha}$ e $\Phi_{r \beta}$ é possível obter todas as variáveis do sistema síncrono (Lorentz et al., 1994).

Na determinação da velocidade considerou-se variação lenta de velocidade, isto é, $\omega_{r(k+1)} \approx \omega_{r(k)}$ (Vas, 1998). Esta aproximação pode ser considerada desde que $\omega_{r}$ não apresente variação significativa entre dois instantes de amostragem; os resultados mostraram, ainda que torque de carga seja aplicado ao eixo do MI, que a estimação de $\omega_{r}$ tem boa precisão.

O motor sob teste é o mesmo que foi utilizado por Valdenebro (2001) e, conseqüentemente, a matriz covariância das pertubações, representada por $Q$, e a matriz covariância das pertubações nas medidas, representada por $R$, são utilizadas neste artigo. São elas:

$$
Q=\left[\begin{array}{ccccc}
0,152 & 0 & 0 & 0 & 0 \\
0 & 0,152 & 0 & 0 & 0 \\
0 & 0 & 0,0457 & 0 & 0 \\
0 & 0 & 0 & 0,0457 & 0 \\
0 & 0 & 0 & 0 & 0,0763
\end{array}\right]
$$

$$
R=0,30518 I_{2 \times 2} \text {. }
$$

\subsection{Lei de controle}

Para obter a lei de controle minimiza-se a seguinte função de custo

$$
J=(\hat{\mathbf{y}}-\mathbf{w})^{T} W_{y}(\hat{\mathbf{y}}-\mathbf{w})+\Delta \mathbf{u} W_{u} \Delta \mathbf{u}
$$

na qual

- $\mathbf{w} \in \Re^{\left(n_{y} q\right) \times 1}$ é o vetor de referências futuras para as saídas a serem controladas;

- $W_{y} \in \Re^{\left(n_{y} q\right) \times\left(n_{y} q\right)}$, matriz definida-positiva, geralmente é diagonal e que permite enfatizar cada uma das saídas controladas e suas predições;

- $W_{u} \in \Re^{n_{u} \times n_{u}}$, matriz definida-positiva, usualmente diagonal e que pondera o esforço de controle das entradas;

- $\Delta \mathbf{u} \in \Re^{n_{u} p \times 1}$ é o vetor incremento de entradas.

Na função custo é assumido que o incremento de controle é nulo após o horizonte de controle e como o modelo é linearizado, obtém-se algebricamente o seu valor mínimo. As matrizes que representam a predição do motor de indução $H^{\prime}, H^{\prime \prime}$ e $H^{\prime \prime \prime}$ - devem ser atualizadas a cada ciclo de controle, pois contém termos variantes no tempo e dependentes dos estados. Ao se minimizar a função dada pela equação (9) obtém-se a seguinte lei de controle:

$$
\Delta \mathbf{u}=\left(H^{\prime \prime T} W_{y} H^{\prime \prime}+W_{u}\right)^{-1} H^{\prime \prime T} W_{y}\left(\mathbf{w}-H^{\prime} \mathbf{x}-H^{\prime \prime \prime} \mathbf{d}\right)
$$

Na determinação da lei de controle, o vetor $\delta$, formado pela soma do termo linearizado com o torque, é considerado constante nos instantes futuros da predição e, conseqüentemente, a obtenção do vetor predição da perturbação, dado por d, torna-se mais simples.

$\mathrm{Na}$ representação por espaço de estado, dada pela equação (6), o torque de carga $T_{c}$ é tratado como uma perturbação externa. Como ele se relaciona com os estados da máquina e estes estados são conhecidos $-i_{s d}$ e $i_{s q}$ são medidos e $\Phi_{r d}$ e $\omega_{r}$ são estimados)-, o seu valor pode ser obtido a partir da equação eletromecânica do motor escrita na sua forma discreta. Esta equação, que descreve a dinâmica da velocidade da máquina, é dada por

$$
T_{c(k)}=\frac{3 p}{2} \frac{L_{m}}{L_{r}} i_{s q(k)} \Phi_{r d(k)}-\frac{J_{t}}{p} \frac{\omega_{r(k+1)}-\omega_{r(k)}}{T_{a}}
$$

Nota-se nesta equação que o torque de carga depende da magnitude da inércia do acoplamento motor-carga; entretanto, neste artigo o valor da inércia da carga é admitido 
desconhecido. Na aplicação desta equação para o cálculo do torque, a inércia total das partes girantes é feita igual à inércia do motor. Os resultados apresentados posteriormente mostram que esta suposição é válida, desde que o valor da inércia da carga não seja muito maior que a do motor.

A presença da velocidade síncrona $\omega_{s}$ no modelo de estados torna o sistema, que já é não linear, variante no tempo. Considerando que $\omega_{e}$ é a velocidade de escorregamento e que a orientação pelo fluxo do rotor é utilizada, $\omega_{s}$ pode ser obtida através da seguinte equação;

$$
\omega_{s}=\omega_{r}+\omega_{e}=\omega_{r}+\frac{L_{m}}{\tau_{r}} \frac{i_{s q}}{\Phi_{r d}}
$$

O diagrama de bloco do sistema, que utiliza algoritmo preditivo aplicado ao controle de $\Phi_{r}$ e $\omega_{r}$ do MI, está mostrado na Figura 1.

\section{RESULTADOS}

Os dados do motor de indução sob teste estão colocados no Apêndice A; a carga é um gerador de corrente contínua, cuja inércia é aproximadamente 20 vezes a inércia do motor. $\mathrm{O}$ atrito viscoso foi desprezado porque o motor de indução sob teste não tem ventilador acoplado ao seu eixo. Os algoritmos de estimação, a conversão de variáveis $\alpha \beta \Leftrightarrow d-q$, a medição de velocidade, a modulação Space Vector e o controle MBPC linearizado foram implementados no kit de desenvolvimento TMS320F2812 baseado em DSP. Antes dos testes experimentais foram feitas simulações para a sintonia dos algoritmos empregados no acionamento.

\subsection{Testes de sintonia dos algoritmos}

Para uma sintonia do algoritmo do MBPC e do filtro de Kalman utilizou-se o programa comercial Simulink/Matlab, sendo os parâmetros do MI dados no Apêndice A. Na aplicação do MBPC utilizou-se o modelo linearizado, sendo os sinais de controle dados pelos acréscimos de tensão $\Delta V_{s d}$ e $\Delta V_{s q}$, que são adicionados aos atuais valores de tensão de eixos direto $\left(V_{s d}\right)$ e em quadratura $\left(V_{s q}\right)$, respectivamente

A escolha dos horizontes de predição $\left(n_{y}\right)$ e de controle $\left(n_{u}\right)$ tem efeito imediato sobre a ordem das matrizes $H^{\prime}$, $H^{\prime \prime}$ e $H^{\prime \prime \prime}$; se eles são altos, a ordem das matrizes aumenta, aumentando, assim, o tempo para executar o algoritmo do MBPC. Após várias simulações obteve-se uma sintonia adequada com $n_{y}=2$ e $n_{u}=1$ (de Santana, 2007). Com estes valores, o tempo demandado para rodar o algoritmo do MBPC foi $20 \mu \mathrm{s}$. Como se observa, este valor é baixo, pois além de $n_{y}$ e $n_{y}$ serem pequenos, as inversões e multiplicação de matrizes são feitas a priori. O tempo de controle foi $6 \mathrm{~ms}$, que é significativamente menor que as constantes de tempo relacionadas ao motor e, conseqüentemente, permite a sua aplicação em tempo real. A matriz que pondera as saídas controladas é a matriz identidade $W_{y}=I_{4 \times 4}$ e aquela que pondera o esforço de controle é dada por

$$
W_{u}=\left[\begin{array}{cc}
W_{u / V_{s d}} & 0 \\
0 & W_{u / V_{s q}}
\end{array}\right]=\left[\begin{array}{cc}
0,15 & 0 \\
0 & 1
\end{array}\right]
$$

na qual

- $W_{u / V_{s d}}$ é o valor que pondera o esforço de controle da entrada $\Delta V_{s d} \mathrm{e}$

- $W_{u / V_{s q}}$ é o valor que pondera o esforço de controle da entrada $\Delta V_{s q}$.

Os resultados de simulação estão colocados na Figura 2. O resultado do teste mostrado na Figura 2(a) refere-se a um perfil trapezoidal de velocidade com reversão para uma carga cuja torque é proporcional ao quadrado da velocidade e sua inércia é o dobro da inércia do motor, sendo que o valor de seu torque, quando o eixo do motor atinge a velocidade de $600 \mathrm{rpm}$, é o seu valor nominal de $12,3 \mathrm{~N} \cdot \mathrm{m}$. Cuidados foram tomados na simulação para que o torque de carga $T_{c}$ seja sempre frenante, o que significa inverter o seu sinal, toda vez que a velocidade se inverte. Saliente-se que os modelos empregados no controlador e no FKE consideram apenas a inércia do motor, ignorando, portanto, a inércia da carga. Observa-se na Figura 2(a) que apesar de o torque de carga ser nominal, a regulação da velocidade em regime permanente é satisfatória. Em um segundo teste é acoplada abruptamente uma carga que demanda o torque nominal do motor, que é igual a $12,3 \mathrm{~N} . \mathrm{m}$ ), e cuja inércia é o dobro da inércia do motor. Como se observa na Figura 2(b), a queda de velocidade devido à entrada da carga é de aproximadamente $15 \%$ em relação a referência. Caso fosse possível prever a entrada da carga, o desempenho do controlador seria melhor, pois seriam gerados sinais de controle que aumentariam a velocidade antes da entrada da carga, minimizando, então, a variação da velocidade.

\subsection{Testes experimentais}

O período de amostragem das tensões e das correntes, bem como o período de chaveamento do inversor foi $300 \mu \mathrm{s}$. Embora no controle de velocidade de motores de pequena potência seja usual operar com freqüências de chaveamento da ordem de $10 \mathrm{kHz}$, escolheu-se o valor de $3,3 \mathrm{kHz}$ pela conveniência de se calcular os estados com o FKE e executar o algoritmo de modulação Space Vector na mesma interrupção, diminuindo o custo computacional e a complexidade da implementação.

Foram duas as interrupções utilizadas pelo $D S P$ para executar o controle proposto: 


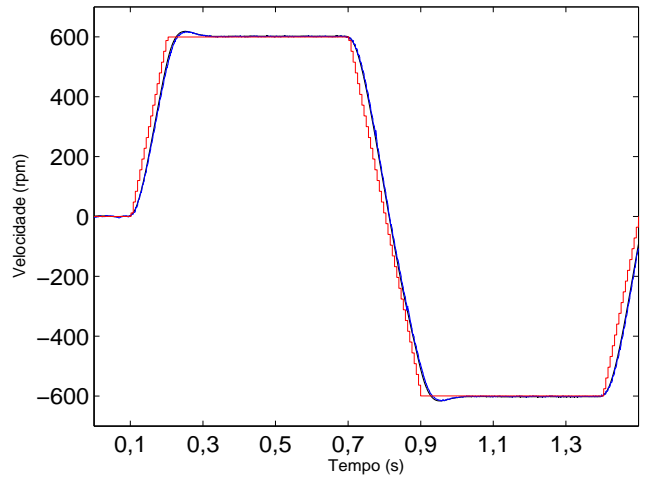

(a) Perfil trapezoidal de $\pm 600 \mathrm{rpm}$, com carga proporcional ao quadrado da velocidade

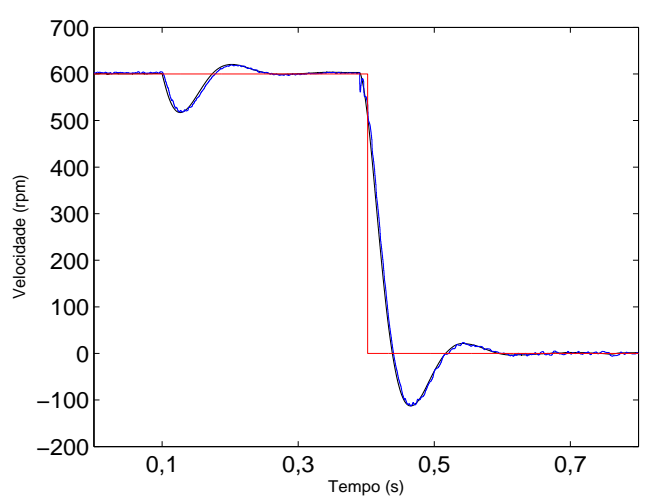

(b) Perfil retangular de $600 \mathrm{rpm}$ a $0 \mathrm{rpm}$, com carga nominal de 12,3 N.m aplicada ao eixo no instante $t=0,1 \mathrm{~s}$

Figura 2: Variações trapezoidal e retangular de velocidade.

1. A interrupção da amostragem inicia-se com a medição dos valores de tensões de linha e das correntes de estator que são, inicialmente, representadas em ponto flutuante e, posteriormente, descritas nas coordenadas estacionárias $\alpha \beta$. O período de amostragem dessas medições é $300 \mu s$. Este valores de tensão e correntes são entradas para o FKE que estima $\Phi_{r \alpha}, \Phi_{r \beta}$ e $\omega_{r}$ a cada período de $300 \mu s$, que convertidos ao sistema síncrono (coordenadas $d-q$ ) são disponibilizados ao algoritmo MBPC. Ainda nesta interrupção, os sinais de controle $V_{s d}$ e $V_{s q}$ a serem aplicados ao motor são convertidos às coordenadas $\alpha-\beta$ e utilizadas pelo algoritmo Space Vector Modulation, que tem a função de gerenciar a sequiência de chaveamento dos IGBT's do inversor.

2. Esta interrupção inicia-se com a medição da velocidade do motor, que é utilizada apenas para verificar o desempenho da respectiva velocidade estimada pelo FKE. Na seqüência o algoritmo MBPC é executado com suas saídas dadas pelos acréscimos de tensão de eixos direto e em quadratura, que devem ser somados ao valor anterior da tensão. Estes valores são disponibilizados na memória para serem empregados pela interrupção anterior, que é responsável pela conversão das tensões ao sistema estacionário e, dessa maneira, realizar a tarefa do SVM. A cada 6 ms o algoritmo de controle gera uma nova lei de controle e as entradas calculadas são aplicadas nos terminais do motor. Como o período de estimação do FKE é $300 \mu \mathrm{s}$, a cada ciclo do algoritmo de controle corresponde 20 estimativas do fluxo e da velocidade, embora somente a última seja empregada.

As formas de onda, obtidas experimentalmente, da velocidade, das correntes e dos fluxos apresentaram picos, que são provocados pelo chaveamento do capacitor presente no conversor A/D. Os seguintes testes experimentais foram realizados:

\section{Variação de velocidade com reversão:}

Com formas de onda de perfil degrau e trapezoidal os resultados estão colocados, respectivamente, nas Figuras 3(a) e 3(b), sendo que para cada teste são apresentadas as velocidades medida e estimada. Embora nos dois testes a medição da velocidade foi altamente afetada por ruídos, que provocaram a distorção dos pulsos gerados pelo encoder, os resultados obtidos foram satisfatórios, não apresentando desvios significativos em relação a referência, seja ela degrau ou trapezoidal.

Com o objetivo de se verificar o desempenho do controle para velocidades baixas, foi testada também a reversão de velocidade de $150 \mathrm{rpm}$ a $-150 \mathrm{rpm}$ com o motor a vazio, e o resultado está colocado na Figura 3(c). Abaixo deste valor, os resultados pioraram muito, tanto no regime permanente quanto no regime dinâmico. Um dos motivos desta deterioração é a diminuição da relação entre o sinal de controle e o ruído, porque os sinais de tensão do estator diminuem à medida que a velocidade controlada diminui.

Variação de velocidade sem reversão e com o motor a vazio:

As Figuras 4(a) e 4(b) mostram, respectivamente, o comportamento das velocidades de referência, a medida e a estimada com variação abrupta de 0 rpm a 300 rpm e com variação trapezoidal de $0 \mathrm{rpm}$ a $450 \mathrm{rpm}$, com tempos de subida de $t=300 \mathrm{~ms}$.

Variação de fluxo de rotor e com o motor a vazio: 


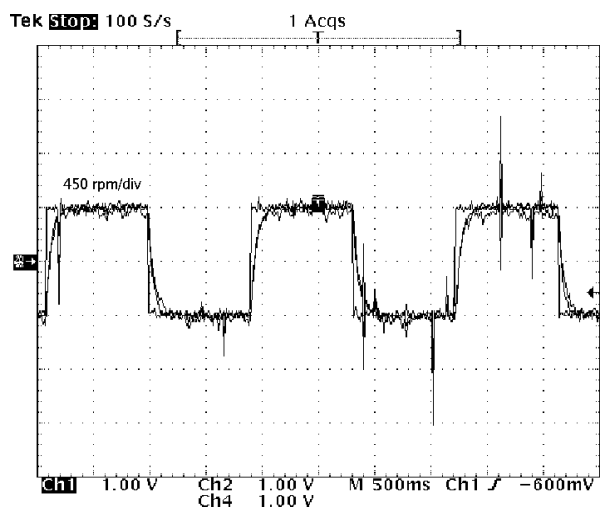

(a) Variação abrupta de $450 \mathrm{rpm}$ a $-450 \mathrm{rpm}$.

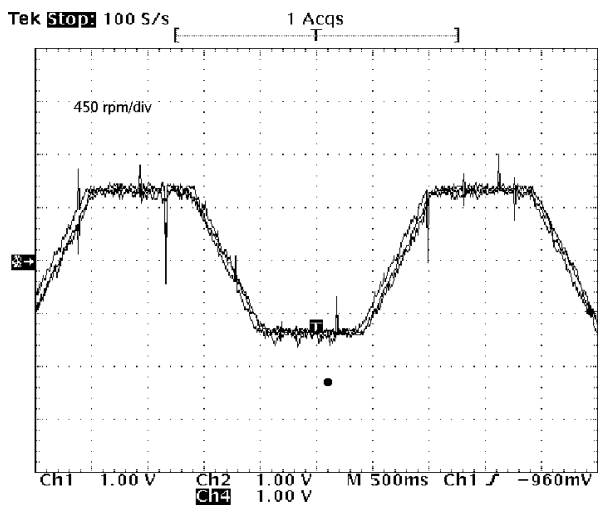

(b) Variação trapezoidal de $600 \mathrm{rpm}$ a $-600 \mathrm{rpm}$.

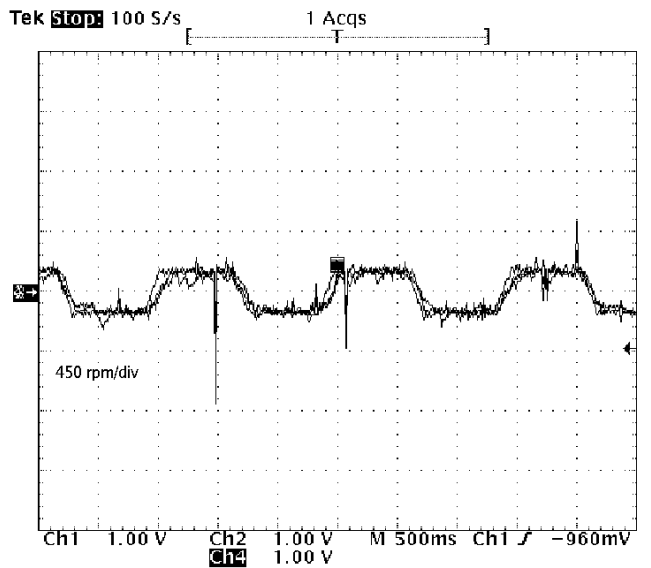

(c) Variação trapezoidal: $\pm 150 \mathrm{rpm}$ em $120 \mathrm{~ms}$.

Figura 3: Motor sem carga: variação de velocidade com reversão.

A Figura 5 mostra nos canais 2 e 4 os valores de referência e estimado do fluxo do rotor, respectivamente; a velocidade estimada do eixo, cujo valor de referência é $450 \mathrm{rpm}$, é a do canal 1. Como se observa, o fluxo estimado segue o da referência e a velocidade é mantida, ainda que o fluxo varie.

\section{Teste com carga:}

$\mathrm{Na}$ sintonia por simulação do controlador preditivo considerou-se que a inércia da carga mecânica é o dobro da inércia do motor. Contudo, nos testes experimentais, o gerador de corrente contínua tem inércia aproximada de 20 vezes a do motor e os resultados obtidos com a sintonia original mostraram que o desempenho do MBPC não foi adequado, quando o motor foi submetido a mudanças significativas de carga. Sendo assim, foi necessário obter nova sintonia do MBPC para que o sistema tivesse bom desempenho com o novo momento de inércia. As novas matrizes de sintonia obtidas foram

$$
W_{u}=I_{2 \times 2} e W_{y}=\left[\begin{array}{cccc}
0,5 & 0 & 0 & 0 \\
0 & 10 & 0 & 0 \\
0 & 0 & 0,5 & 0 \\
0 & 0 & 0 & 10
\end{array}\right]
$$

O desempenho do acionamento é visto na Figura 6, para a aplicação de um torque de carga de $10,36 N \cdot m$, quando o motor funcionava a vazio com velocidade do eixo igual a

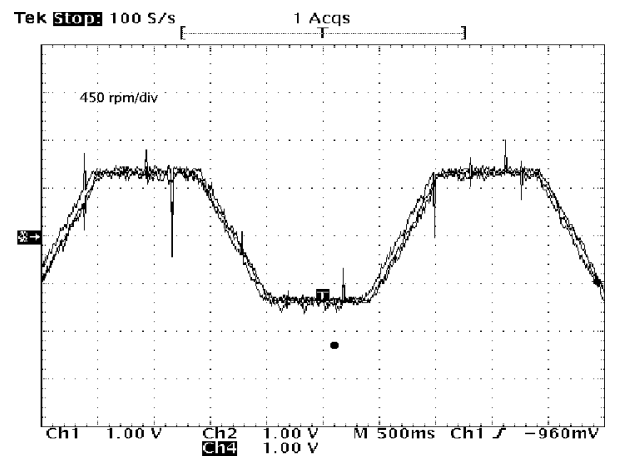

(a) Variação abrupta de 0 a 300 rpm

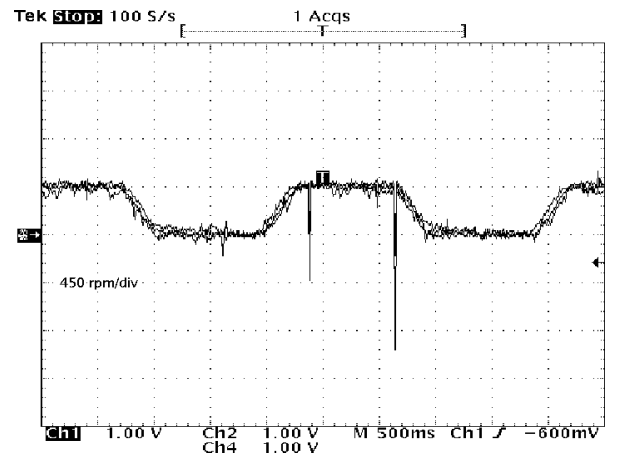

(b) Variação trapezoidal de 0 a 450 rpm

Figura 4: Motor sem carga: variação de velocidade sem reversão. 


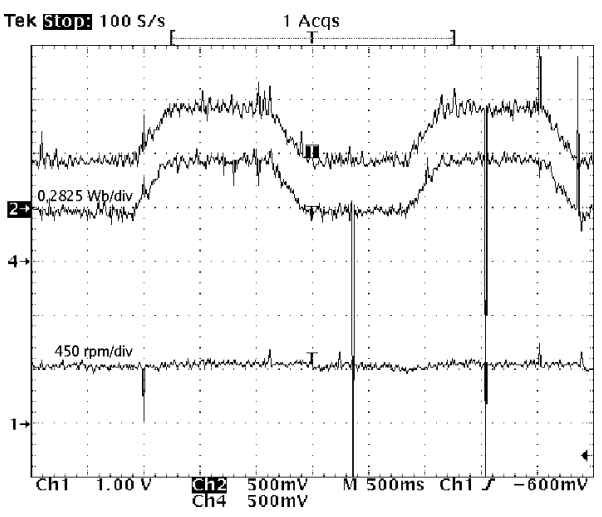

Figura 5: Variação de fluxo de rotor do valor nominal a 50\% do valor nominal em $300 \mathrm{~ms}$ e com o motor a vazio.

$600 \mathrm{rpm}$. A corrente $i_{s d}$ mostrada pelo canal 2 mantém seu valor constante, o que evidencia que o fluxo do rotor também tem sua magnitude constante e que a orientação de fluxo do rotor é mantida. Também nota-se que a magnitude da corrente $i_{s q}$, que é diretamente proporcional ao torque eletromecânico desenvolvido pelo motor, é aumentada no instante em que a carga é aplicada ao motor; uma vez que o fluxo de rotor é constante, este aumento está de acordo com a teoria da orientação do fluxo de rotor. A resposta dinâmica da velocidade foi satisfatória, apresentando um erro de regime de cerca de $3 \%$, o que é aceitável na maioria das aplicações e, por esta razão, não foi realizado uma nova sintonia do controlador.

\section{CONCLUSÃO}

Neste artigo foi apresentada a aplicação de um controlador preditivo multivariável utilizando um modelo de estado para representar motor de indução. Os estados foram estimados

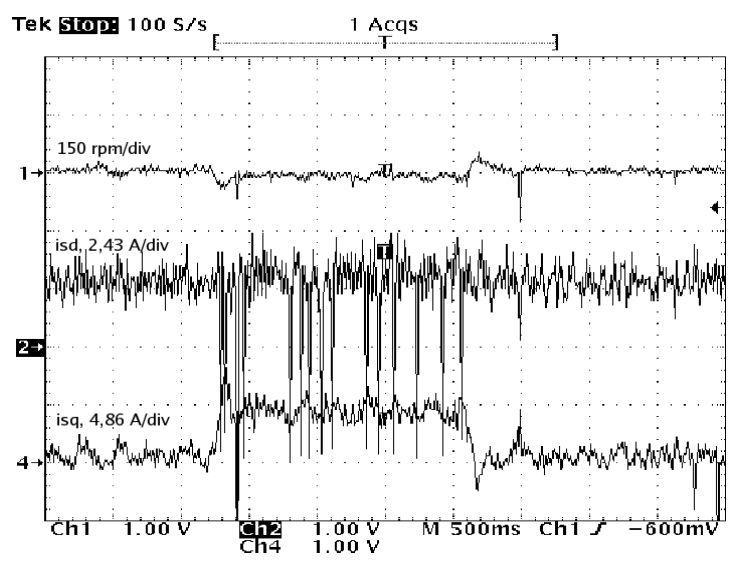

Figura 6: Teste com carga de $10,36 N \cdot m$ : velocidade estimada (canal 1), $i_{s d}$ (canal 2) e $i_{s q}$ (canal 4). empregando um filtro de Kalman estendido. O sistema de acionamento utilizou a orientação de fluxo de rotor e o torque de carga foi considerado como uma perturbação, sendo a sua magnitude estimada pelo uso da equação de movimento do motor. Na execução do algoritmo MBPC, o valor da inércia da carga não foi utilizado. Os resultados de simulação e experimentais mostraram que é possível com a abordagem multivariável controlar o fluxo e a velocidade através da manipulação direta das tensões de estator, sem a necessidade de controladores de corrente.

Dentre as características positivas do algoritmo MBPC aplicado ao acionamento do motor de indução destaca-se o fato de não serem necessários ciclos de controle pequenos, diferentemente do que ocorre na maioria das estratégias de controle. Recentemente, são encontrados na literatura controladores PI que atuam com ciclo de controle em torno de $100 \mu s$, enquanto que neste trabalho, operou-se com um ciclo bem maior, isto é, cerca de $6 \mathrm{~ms}$, dando uma grande margem de folga para que o DSP possa executar outras tarefas que exijam, por exemplo, antecipações com horizontes maiores.

Embora a montagem utilizada nos experimentos tenha apresentado ruídos significativos, notadamente para velocidades baixas, pode-se afirmar que os resultados obtidos em tempo real mostram a viabilidade e o grande potencial de uso do MBPC em tempo real no acionamento elétrico, encorajando futuros estudos.

\section{A DADOS DO MOTOR}

220/380 V , 8, 4/4,86 A, $60 \mathrm{~Hz}, 3 \mathrm{HP}, 1730 \mathrm{rpm}, 4$ pólos, $R_{s}=2,229 \Omega, R_{r}=1,522 \Omega, L_{m}=0,23848 \mathrm{H}, L_{s}=$ $0,2448 \mathrm{H}, L_{r}=0,24971 \mathrm{H}, L_{s}^{\prime}=0,0192 \mathrm{H}, L_{r}^{\prime}=0,0194$ $\mathrm{H}, \tau_{s}^{\prime}=0,0086 s$ e $\tau_{r}^{\prime}=0,0128 s$.

\section{AGRADECIMENTOS}

Os autores agradecem à FAPESP, à CAPES e ao CNPq pelo suporte financeiro dado ao desenvolvimento desta pesquisa.

\section{REFERÊNCIAS}

Altuna, J. A. T. (2002). Análise dinâmica de controladores de corrente para máquinas de indução trifásicas alimentadas por inversor PWM, Tese de doutorado, Universidade Estadual de Campinas.

de Santana, E. S. (2007). Algoritmo Preditivo Baseado no Modelo Aplicadao ao Controle de Velocidade do Motor de Indução, Tese de doutorado, Universidade Estadual de Campinas. 
Hedjar, R., Toume, R., Boucher, P. and Dumur, D. (2004). End poit constraints nonlinear predictive control with integral action for induction motor, Proceedings of the 2004 IEEE International Conference on Control Applications, Vol. 1, pp. 1691-1696.

Kennel, R. and Linder, A. (2005a). Direct model predictive control- a new direct predictive control strategy for electrical drives, Power Electronics and Applications, European Conference on p. 10pp.

Kennel, R. and Linder, A. (2005b). Model predictive conmtrol for electrical drives, Power Electronics Specialists Conference-PESC pp. 1793-11799.

Kennel, R., Linder, A. and Linke, M. (1997). Generalized predictive control (GPC)-ready for use in drive applications?, Power Electronics Specialists Conference, 2001. PESC. 2001 IEEE 32nd Annual, Vol. 4, pp. 1839-1844.

Lorentz, R. D., Lipo, T. A. and Novotny, D. W. (1994). Motion control with induction motors, Proceeding of the IEEE-Special issue on power electronics and motion control pp. 1215-1240.

Low, K. S., Chiun, K. Y. and Ling, K. V. (1997). A dsp-based servo system using generalized predictive control, Proceedings of the Power Conversion Conference, Vol. 1, pp. 507-512.

Moreira. V.D., P.A. Ferreira, W. A. (2004). Controle preditivo robusto de sistemas híbridos incertos, XV Congresso Brasileiro de Automática, Gramado, Vol. CDROM.

Rossiter, J. A. (2003). Model Based Predictive Control - A Practical Approach, CRC Press.

Valdenebro, L. R. (2001). Observadores adaptativos de fluxo e velocidade para motores de indução - estudo e implementação, Tese de doutorado, Universidade Estadual de Campinas.

Vas, P. (1998). Sensorless vector and direct torque control, Oxford science publications.

Zhang, L., Norman, R. and Shepherd, W. (1997). Longrange predictive control of current regulated pwm for induction motor drives using the synchronous reference frame, IEEE Transactions on control systems techno$\operatorname{logy}$ 5(1): 119-125. 